\title{
Reviewers Index
}

(The reviewers who completed the first peer-review for manuscripts submitted during Nov 1, 2019-Oct 31, 2020.)

The ten reviewers written in boldface reviewed more than 6 papers.

Annals of Vascular Diseases do thank all the reviewers for their strong support and cooperation.

\begin{tabular}{|c|c|c|c|}
\hline $\mathbf{A}$ & Hiraoka, Arudo & Kokubo, Taku & $\mathbf{N}$ \\
\hline Adachi, Koichi & Hiromatsu, Shinichi & Komai, Hiroyoshi & Nakamura, Takashi \\
\hline Akahane, Masaaki & Hosaka, Akihiro & Kondo, Norihiro & Nakazawa, Tatsu \\
\hline Akazawa, Hiroshi & Hoshina, Katsuyuki & Kritayakirana, Kritaya & Nishibe, Toshiya \\
\hline Akutsu, Koichi & Hosoi, Yutaka & Kudo, Toshifumi & Nishigami, Kazuhiro \\
\hline Al-Salman, Mussaad M.S. & Huh, Seung & Kuma, Sosei & Nishimura, Motonobu \\
\hline Altarazi, Louay & & Kunihara, Takashi & Nishimura, Yoshiharu \\
\hline Anai, Hiroshi & $\mathbf{I}$ & Kurabayashi, Masahiko & Nunokawa, Masao \\
\hline Asakura, Toshihisa & Ichihashi, Shigeo & Kurihara, Nobuhisa & \\
\hline Asano, Soichi & Inaba, Masashi & Kurimoto, Yoshihiko & $\mathbf{O}$ \\
\hline \multirow[t]{2}{*}{ Azuma, Nobuyoshi } & Inoue, Masanori & & Obara, Hideaki \\
\hline & Inuzuka, Kazunori & $\mathbf{L}$ & Ogawa, Tomohiro \\
\hline B & Ishibashi, Hiroyuki & Laohapensang, Kamphol & Ogawa, Yukihisa \\
\hline Banno, Hiroshi & Ishikawa, Tetsuya & Lee, Byung-Boong & Ohhashi, Toshio \\
\hline Bashar, Abul Hasan & Ito, Hiroyuki & & Ojiro, Masataka \\
\hline Muhammad & Ito, Tsutomu & $\mathbf{M}$ & Okazaki, Jin \\
\hline \multirow[t]{2}{*}{ Bessho, Ryuzo } & Iwata, Hirohide & Maeda, Hideaki & Orihashi, Kazumasa \\
\hline & Iyori, Keiji & Maeda, Koji & Otani, Norifumi \\
\hline $\mathbf{C}$ & Izumi, Yuichi & Masaki, Hisao & \\
\hline Cheng, Stephen WK & & Matsubara, Junichi & $\mathbf{P}$ \\
\hline \multirow[t]{2}{*}{ Chikazawa, Genta } & $\mathbf{J}$ & Matsubara, Kentaro & Pinjala, Ramakrishna \\
\hline & Jibiki, Masatoshi & Matsubara, Shinobu & \\
\hline $\mathbf{F}$ & Jin, Hyun Joh & Matsuda, Hitoshi & $\mathbf{S}$ \\
\hline Fletcher, John & & Matsumoto, Takuya & Saiga, Atsushi \\
\hline Fujii, Takeshiro & $\mathbf{K}$ & Matsushita, Masahiro & Sakuda, Hitoshi \\
\hline Fukuda, Hirotsugu & Kadohama, Takayuki & Midorikawa, Hirofumi & Sano, Masaki \\
\hline Fukuda, Ikuo & Kamal, Dhafer M & Minatoya, Kenji & Sato, Osamu \\
\hline Fukui, Kozo & Kamiya, Hiroyuki & Mine, Takahiko & Sato, Tadaya \\
\hline \multirow[t]{2}{*}{ Furuyama, Tadashi } & Kanaoka, Yuji & Mitsuoka, Hiroshi & Satokawa, Hirono \\
\hline & Katsumata, Takahiro & Miyata, Tetsuro & Sawano, Makoto \\
\hline $\mathbf{H}$ & Kawaharada, Nobuyoshi & Mo, Makoto & Seto, Tatsuichiro \\
\hline Hamamoto, Masaki & Kichikawa, Kimihiko & Morikage, Noriyasu & Shigematsu, Kunihiro \\
\hline Handa, Nobuhiro & Kitagawa, Takeshi & Morota, Tetsuro & Shiiya, Norihiko \\
\hline Harada, Hirohisa & Kitano, Ikurou & Motomura, Noboru & Shimizu, Tsuyoshi \\
\hline Haruta, Naoki & Kobayashi, Masayoshi & Murakami, Atsubumi & Shimpo, Hideto \\
\hline Hattori, Tsutomu & Kodama, Akio & & Shindo, Shunya \\
\hline Hayashida, Naoki & Koizumi, Jun & & Sugano, Norihide \\
\hline
\end{tabular}

(cc) BY-NC-SA (02020 The Editorial Committee of Annals of Vascular Diseases. This article is distributed under the terms of the Creative Commons Attribution License, which permits use, distribution, and reproduction in any medium, provided the credit of the original work, a link to the license, and indication of any change are properly given, and the original work is not used for commercial purposes. Remixed or transformed contributions must be distributed under the same license as the original. 
Suzuki, Hiroshi

Suzuki, Shinichi

$\mathbf{T}$

Taguchi, Shinichi Tajima, Hiroyuki

Takagi, Gen

Takagi, Yasushi

Takahashi, Toshiki

Takano, Hiroshi

Takayama, Katsutoshi
Takayama, Toshio Takayama, Yutaka Tanaka, Hiroyuki Tanemoto, Kazuo Tateno, Kaoru Tomaru, Takanobu Toya, Naoki

Tsukube, Takuro

$\mathbf{U}$

Ueda, Tatsuo
Ueyama, Keishi

W

Wada, Hideichi

Wang, Jinsong

Washiyama, Naoki

Y

Yamamoto, Hiroshi

Yamamoto, Kota

Yasugi, Takumi
Yiu, Wai-ki

Yoshida, Hiroki

Yoshimura, Koichi

Yoshitake, Akihiro

Yoshizumi, Masao

Yunoki, Yasuhiro

Yuri, Koichi

Z

Zempo, Nobuya 